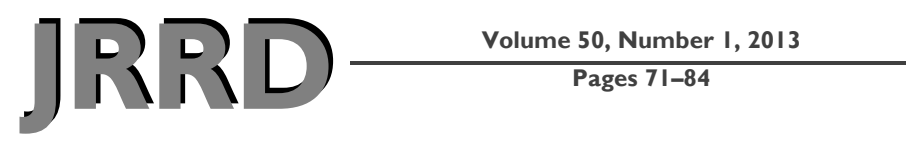

\title{
Compensatory arm reaching strategies after stroke: Induced position analysis
}

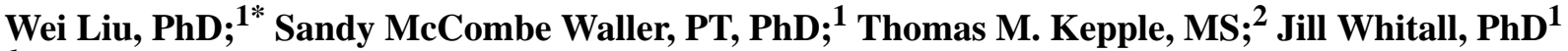 \\ ${ }^{1}$ Department of Physical Therapy and Rehabilitation Science, School of Medicine, University of Maryland, Baltimore, \\ $M D ;{ }^{2}$ C-Motion Inc, Germantown, MD
}

\begin{abstract}
After stroke, movement patterns of the upper limb (UL) during functional arm reaching change to accommodate altered constraints. These compensatory movement control strategies do not, however, have a one-to-one mapping with posttraining outcomes. In this study, we quantify arm movement control strategies in unilateral and bilateral reaching tasks using induced position analysis. In addition, we assess how those strategies are associated with UL residual impairments and with functional improvement after a specific bilateral arm training intervention. Twelve individuals with chronic stroke were measured while reaching to a box as part of their pre- and posttesting assessments. Other measurements included the Fugl-Meyer Upper Extremity Assessment (FM), Modified Wolf Motor Function Test (WT), and the University of Maryland Arm Questionnaire for Stroke (UMAQS). We identified arm control strategies that did not differ between unilateral and bilateral tasks but did differ by FM impairment level and by predicted gains in WT but not UMAQS. Increased shoulder relative to elbow moment contribution was associated with less impairment and greater gains of speed in functional tasks. These results suggest that one goal of training to achieve better outcomes may be to decrease the abnormal coupling of the shoulder and elbow.
\end{abstract}

Key words: arm reaching, compensatory strategies, couple dynamics, functional recovery, induced position analysis, motor function, multijoint control, rehabilitation, stroke, upper limb.

\section{INTRODUCTION}

Stroke is the leading cause of adult disability in the United States, with 5.5 million people affected by stroke and rising [1]. More than 750,000 Americans are affected by a new or recurrent stroke each year, with 95 percent of stroke survivors having some upper-limb (UL) dysfunction in routine daily activities, including dressing, bathing, self-care, and writing [2]. Twenty percent of those with stroke regain no functional use of their hemiparetic arm [3]. Several rehabilitation interventions, such as constraint-induced therapy [4], robotics training [5-6], and bilateral arm training, have demonstrated some recovery of UL function in chronic stroke survivors [7-8]. Which stroke survivor can benefit from which training, however,

\footnotetext{
Abbreviations: $3-\mathrm{D}=$ three-dimensional, $\mathrm{BATRAC}=$ Bilateral Arm Training with Rhythmic Auditory Cueing, CNS = central nervous system, FM = Fugl-Meyer Upper Extremity Assessment, IAA = induced acceleration analysis, IPA = induced position analysis, PI = principal investigator, UL = upper limb, UMAQS = University of Maryland Arm Questionnaire for Stroke, WT $=$ Modified Wolf Motor Function Test.

*Address all correspondence to Wei Liu, PhD; Department of Physical Therapy, University of South Alabama, $307 \mathrm{~N}$ University Blvd, HAHN Rm \# 2011, Mobile, AL 36688; 251445-9247; fax: 251-445-9238. Email: liuw77@gmail.com http://dx.doi.org/10.1682/JRRD.2011.04.0074
} 
is not clear because there is not a one-to-one relationship between residual movement control strategies and posttraining outcomes [9]. In this study, we initially focused on determining arm movement control strategies in stroke for unilateral and bilateral tasks and subsequently on exploring how those strategies are associated with both residual impairment levels and with functional improvements after a specific bilateral arm training intervention.

After stroke, movement patterns likely change to accommodate altered constraints such as hypertonicity, extensibility limitations, and reduced cortical input in order to accomplish UL functions such as arm reaching. This new movement pattern is termed a compensatory movement control strategy. In early clinical observations, a qualitative description of compensatory movement strategies was used to categorize pathological synergies, which included the flexor synergy (shoulder flexion, shoulder abduction, elbow flexion, forearm supination) and the extensor synergy (shoulder extension, shoulder adduction, elbow extension, forearm pronation) [10]. More recently, a functional quantitative analysis of UL movement during arm reaching illustrated that patients with hemiparesis used more trunk flexion and shoulder abduction to compensate for reduced elbow extension [11-12] and demonstrated abnormal shoulder abductor power to compensate for reduced shoulder flexor power [13]. These compensatory movement strategies are evident during unilateral UL tasks.

In daily life, however, bimanual actions are common motor tasks and are arguably more important poststroke for individuals to recover than unilateral actions alone. Therefore, it is important to study bilateral as well as unilateral tasks. Moreover, there may be advantages of performing certain tasks bilaterally. For example, when nondisabled individuals move both arms simultaneously to targets at different distances, the arms are temporally and spatially coupled [14-15]. This suggests that the central nervous system (CNS) controls bilateral limb coordination as one unit rather than each limb independently. This coupling phenomenon may have positive consequences for individuals with stroke and provides a rationale for exploring such consequences.

Studies investigating the possibility of UL interlimb coupling in individuals with poststroke hemiparesis have indicated immediate coupling advantages. For example, peak acceleration of the paretic arm during bilateral reaching increases compared with unilateral reaching alone [16-17]. These studies used an end-point hand tra- jectory as the dependent variable, but this leaves some uncertainty as to whether a more proximal joint such as elbow or shoulder can contribute or play a different role during a bilateral versus a unilateral reaching task. In this study, we explore whether movement control strategies are different between unilateral and bilateral tasks and whether they can be distinguished using a contemporary quantitative approach.

Traditional methods to quantify a compensatory movement strategy include kinematic analysis and an inverse dynamics approach [11-13]. UL functional motor tasks, including arm reaching, require simultaneous coordination of wrist, elbow, and shoulder joints, and it is clear that the CNS must manage kinetic factors to achieve the kinematics related to the desired task outcome. Importantly, the net moment at a single joint can produce accelerations at all other joints [18]. This raises a computational difficulty for using inverse dynamics to determine the resulting motion (kinematics) from the causative moment (kinetics) because inverse dynamics takes the observed motion as input to estimate the net joint moment separately from distal joint to proximal joint. Because of this computational difficulty, Lang and Beebe attempted to examine the UL segment contribution to hand function in stroke by isolating one segment and keeping other segments still [19]. While this approach has merit, it does not capture the complete multijoint control strategy during a dynamic functional reaching task in which all segments are moving in an unconstrained manner.

Recently, Kepple et al. used induced acceleration analysis (IAA) [20] based on coupled dynamics principles to examine the individual available movement control resources contribution during gait [21]. He found that ankle plantar-flexor moment provided most of the vertical support during single-limb stance and was the largest source of forward progression, with significant contributions from the knee extensor moment as well [20]. Later, IAA was extended into induced position analysis (IPA) to determine the contribution of individual muscles from knee flexion at toe off to peak knee flexion during the swing phase of gait [22]. The techniques of IAA and IPA allow us to identify the biomechanical factors contributing to movement by directly quantifying the relationships between net joint moment and observed motion. In a previous study of nondisabled individuals, this approach illustrated that shoulder and elbow moments acted together with an "overshoot" and "undershoot" pattern to move the arm forward into the final position [23]. That is, there were minimal contributions from the wrist or 
gravity. The shoulder and elbow acted in opposite directions, with the shoulder acting in a forward direction and the elbow acting as a braking mechanism counterbalancing the shoulder to attain the final position.

To our knowledge, this approach has not been used to quantify compensatory movement control strategies in individuals with stroke. In this study, we had three aims. The first aim was to determine the compensatory movement strategies for unilateral and bilateral reaching in stroke by using IPA. The second aim was to explore whether the identified compensatory movement strategies correlate with residual impairment levels. This would provide evidence that the strategies were meaningful and related to a behavioral set of observations. According to the most recent review article [24], the best predictors of residual UL motor function recovery were initial neurophysiologic measures such as motor-evoked potential amplitude and latency. The most commonly explored predictors were initial motor function measures such as muscle strength and active range of motion. These predictors were mainly tested in acute or subacute stroke survivors because, at the early stage of stroke, these initial measures would have enough sensitivity to predict natural motor function recovery. However, it is also important to understand whether we can predict UL recovery after intervention in the chronic phase of stroke. Therefore, the third aim was to explore whether the compensatory movement strategies correlate with UL recovery response to a specific training, in this case Bilateral Arm Training with Rhythmic Auditory Cueing (BATRAC) [7]. Impairment levels, by themselves, do not always predict either current functional performance or the functional changes after training. Movement strategies may be important predictors of residual or specific intervention outcomes because they give cumulative information on how stroke survivors accomplish the motor task. Cumulative variables that link subcomponents of motor task performance at kinematic (joint angle, etc.) and kinetic (joint moment, etc.) levels may be sensitive to UL motor function recovery. For example, Lang and Beebe found that the active range of motion at all UL segments can predict the hand function variance, but there was no unique contribution pattern by proximal, middle proximal, and distal segments together [19].

The movement strategy that links all segments in a coordinated manner and in a task context such as reaching would represent a multijoint contribution pattern. This pattern should provide a better understanding of how a dynamic functional reaching task is accomplished, and movement strategies that are nearer to nondisabled movement strategies may also be more associated with functional recovery after training in stroke. Therefore, we hypothesized that our compensatory strategies defined through IPA analysis will correlate with residual impairment levels as well as with directional change after a specific intervention.

In summary, we extended the study of compensatory movement strategies by using a coupled dynamics approach of IPA and by comparing these strategies between unilateral and bilateral reaching tasks. Based on previous studies [16-17], we hypothesized that compensatory movement strategies in a bilateral reaching task are different than in a unilateral reaching task. We also explored the correlation of these strategies with residual impairment levels and determined whether they correlate with postintervention changes in motor function when the intervention is BATRAC. We chose BATRAC as an intervention because it takes advantage of the interlimb coupling principle described previously and has shown promise as a viable intervention for those with chronic stroke [7-8].

\section{METHODS}

\section{Participants}

Twelve participants with chronic unilateral ischemic stroke were continuously enrolled from two parent training studies after providing written informed consent approved by both the University of Maryland and Baltimore Department of Veterans Affairs Medical Center Institutional Review Boards. Inclusion criteria were (1) unilateral ischemic or hemorrhagic stroke, (2) aged 30 to $80 \mathrm{yr}$, (3) at least 6 mo poststroke and completion of all conventional inpatient and outpatient therapy, (4) residual use of the paretic arm demonstrated by a minimal ability to initiate antigravity shoulder/elbow movement in flexion and extension in the transverse-sagittal plane, (5) ability to perform 3 in. of forward translation of the hand as a minimum reaching distance, and (6) ability to complete the testing protocol as described in the testing procedures. Exclusion criteria included poorly controlled hypertension ( $>160$ / 100), significant orthopedic and/or chronic pain conditions, untreated poststroke depression, and pulmonary or renal failure. Participants' characteristics are provided in the Table. Participants undertook baseline and posttraining testing of reaching and clinical assessments. 
JRRD, Volume 50, Number 1, 2013

Table.

Stroke participants' characteristics.

\begin{tabular}{|c|c|c|c|c|c|c|c|c|}
\hline Subject & $\begin{array}{c}\text { Baseline } \\
\text { FM }\end{array}$ & $\begin{array}{l}\text { Age } \\
\text { (yr) }\end{array}$ & $\begin{array}{c}\text { Baseline } \\
\text { WT (s) }\end{array}$ & $\begin{array}{c}\text { Post } \\
\text { WT (s) }\end{array}$ & $\begin{array}{l}\text { Baseline } \\
\text { UMAQS }\end{array}$ & $\begin{array}{c}\text { Post } \\
\text { UMAQS }\end{array}$ & CVA & Sex \\
\hline 1 & 6 & 54 & 104.6 & 106.6 & 10 & 10 & Left & Male \\
\hline $2^{*}$ & 12 & 72 & 109.7 & 104.6 & 13 & 14 & Right & Female \\
\hline 3 & 14 & 67 & 106.2 & 106.9 & 24 & 21 & Right & Female \\
\hline $4^{*}$ & 14 & 66 & 76.4 & 64.9 & 17 & 21 & Right & Male \\
\hline 5 & 18 & 36 & 106.7 & 109.3 & 17 & 14 & Left & Female \\
\hline $7^{*}$ & 21 & 61 & 58.0 & 50.2 & 26 & 20 & Right & Female \\
\hline 8 & 23 & 53 & 72.2 & 75.3 & 16 & 17 & Right & Male \\
\hline 9 & 23 & 62 & 86.7 & 86.9 & 20 & 14 & Right & Female \\
\hline $10^{* \dagger}$ & 38 & 53 & 13.8 & 12.4 & 34 & 34 & Left & Male \\
\hline $11^{* \dagger}$ & 53 & 56 & 13.2 & 12.7 & 30 & 40 & Left & Female \\
\hline
\end{tabular}

* Subject had improvement at WT.

${ }^{\dagger}$ Subject had FM score $>25$.

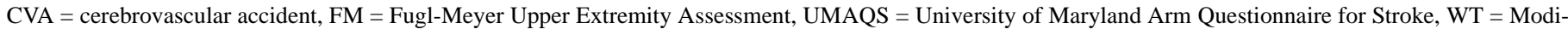
fied Wolf Motor Function Test.

\section{Reaching Experimental Setup}

Arm compensatory strategies were analyzed from unilateral and bilateral reaching at baseline. Participants were seated at a table with an $18 \times 15 \times 9 \mathrm{~cm}$ box in front of them at a distance corresponding to their paretic arm's maximum voluntary reach excursion. The goal of the task was to lift their hand off the table, reach forward with their arm, and contact the lateral aspect of the box in the sagittal plane. Padded straps were used to prevent forward trunk lean and to stabilize the trunk against the back of the chair. The trunk was stabilized to prevent movement because trunk leaning is a typical compensatory strategy during arm reaching [11] and we wanted to eliminate this confounding factor since the training regimen also controlled for movement of the trunk. We note that using this restraint, in fact, means that we were actually investigating compensatory strategies after inhibiting the typical trunk compensation strategy [12]. At the starting position, participants were asked to place their paretic/ nonparetic hand on the surface of the table with palms facing down, $90^{\circ}$ elbow flexion, and shoulder flexion of $0^{\circ}$ as a neutral starting condition. Participants were asked to complete three reaching tasks: unilateral reach with the paretic and nonparetic arms (from the table to the side of the box) and bilateral reaching (simultaneously reaching from the table to the sides of the box with both hands). The bilateral reaching task setup used the box placed in the same location as the unilateral tasks but started with both hands on the table with reaching to the common goal of each side of the box. Participants performed four trials for each condition at preferred speed (Figure 1).

\section{Clinical Assessments}

Baseline and posttraining measures for impairment, function, and disability were also collected for each

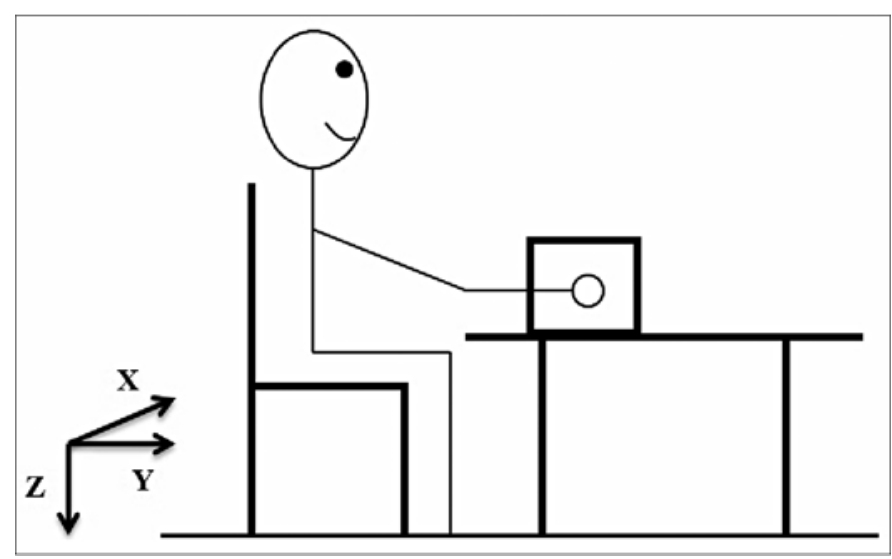

Figure 1.

Schematic diagram of arm reaching in space with orientation of global coordinate systems. $x$-axis is directed laterally to right (flexion/extension axis), $y$-axis is directed anteriorly (abduction/ adduction axis), and $z$-axis is directed superiorly (internal/external rotation axis). 
participant using the Fugl-Meyer Upper Extremity Assessment (FM) [25], the Modified Wolf Motor Function Test (WT) [26], and the University of Maryland Arm Questionnaire for Stroke (UMAQS) [27]. FM measures motor impairment on a 66-point scale. This test has been shown to be valid and reliable in several studies for acute and chronic stroke. WT measures aspects of functional ability while performing 15 UL tasks [28-29]. These measures include performance time, quality of movement, grip strength, and ability to hold a weight. The administration of the test was modified such that use of the nonparetic arm to assist was prohibited in order to assess paretic function alone. This modified test has been reported to have high test-retest reliability and good concurrent validity with FM when used with patients who had mild and moderate hemiparesis [26]. Only the performance time is used in this study. UMAQS measures daily use of the paretic arm. The questionnaire is based on a 5point scale that assesses the degree of independence and use of the paretic limb. It includes unilateral and bilateral tasks as well as considering handedness. This scale is moderately reliable but has not been validated concurrent with other scales [27].

\section{Training}

Each of the 12 participants received 6 weeks of progressive BATRAC training with 3 sessions a week for a total of 18 sessions, each lasting approximately $35 \mathrm{~min}$. Training consisted of four 5 min bouts interspersed with 5 min rest periods. The training device had bilateral handles for grasping, and these were moved along two relatively friction-free tracks that were not connected to one another. The tracks could be adjusted to incline so that arms could move against gravity. Bilateral repetitive arm movements were timed to an auditory metronome set at the participant's preferred speed. There was a stop placed at the maximum excursion of each arm. Participants were encouraged to prioritize keeping to the beat as well as reaching the stop. The stop was progressed for the paretic arm on a sessionby-session basis according to the trainer's perception of patient progress. Unlike the original nonprogressive BATRAC [7-8], in the two parent studies, the speed and incline (arm trajectory increased against gravity) were progressed as well, on a daily basis, based on participant's tolerance and the trainer's judgment.

\section{Data Collection and Reduction for Induced Position Analysis}

A magnetic motion tracking system (Ascension Technology; Burlington, Vermont) was used to record multijoint reaching tasks with a sampling frequency at $50 \mathrm{~Hz}$. Sensors were attached on the dorsum of the right hand, forearm, upper arm, and trunk. Three-dimensional (3-D) sensor data were collected by Motion Monitor Software (Innsport Training, Inc; Chicago, Illinois) and filtered with a fourth-order Butterworth low pass filter (10 Hz cutoff). Each segment's bony landmarks were digitized by stylus to create a local coordinate system. Right-handed coordinate systems were constructed following the convention, with anatomical position being the neutral position. It follows that the $x$-axis is directed laterally to the right, the $y$-axis is directed anteriorly, and the $z$-axis directed superiorly. A customized MATLAB (MathWorks; Natick, Massachusetts) program was used to identify reaching movement onset and offset. Onset of the reaching motion was defined as the time point when position data for the anterior-posterior direction exceeded the mean baseline position (determined over at least 50 samples) plus 5 standard deviations. Reaching movement offset was defined as the time point when the velocity profile fell within the "zero velocity zone," which was defined as the mean baseline velocity (determined over at least 50 samples) plus 5 standard deviations. Dependent variables included the contribution of shoulder moment, elbow moment, wrist moment, and gravity on the hand position trajectory.

\section{Induced Position Analysis}

Liu et al. applied IPA to the same multijoint reaching task described previously [23] in nondisabled adults to determine the hand end-point trajectory contributed by net individual joint moment and gravity. The mathematical details of IPA and the theoretical coupled dynamics principle are explained in detail in the method section of Liu et al. [23]. Briefly, the customized IPA included three major determination steps: (1) 3-D kinematics, (2) 3-D kinetics, and (3) coupled dynamics.

The 3-D segmental Euler kinematics angles $(x-y-z$ order) were computed based on the rotation matrix between global and local coordinate systems of each segment. Consequently, joint angular displacement, velocity, acceleration, and segmental angular velocity were calculated [30]. A traditional inverse dynamics model was used to compute net joint moments. The effect of gravity on the hand was an external force to drive the model. 
Wrist joint force and moment were computed based on the Newton-Euler equations. Following the same principle, elbow force, elbow moment, shoulder force, and shoulder moment were derived. The joint forces and moments were expressed in the local segmental coordinate system.

The customized biomechanical model of a paretic arm was created in SD FAST software (Symbolic Dynamics, Inc; Mountain View, California). The customized biomechanical model of the UL was created in SD FAST software. The model consisted of seven segments: a combined trunk segment and upper arm, forearm, and hand of both limbs with a total of 12 degrees of freedom (2 limbs $\times 6$ degrees of freedom). The wrist joint was modeled as a pin joint (flexion-extension only), the elbow as a universal joint (flexion-extension and radial-ulnar pronation-supination), the shoulder joint as a gimbal joint (flexion-extension, abadduction, and medial-lateral rotation), and the trunk as a ground.

The advantage of IPA is that it can overcome two main limitations of the traditional inverse dynamics method of quantifying arm control strategies. The inverse dynamic approach (1) assumes that the relative contribution of muscular effort is proportional only to the magnitude of the effort and is independent of the position of the body segments, whereas the IPA method is dependent on the position of the body segments, and (2) the inverse dynamics method does not account for the fact that the net moment at a joint will act to accelerate all of the other joints of the body and not just the immediately adjacent joint. IPA quantifies the contribution of multiple-linked joints [18].

Subsequent processing for IPA was computed with customized MATLAB programs. The positions of the model segments were configured on a frame-by-frame basis using data obtained from experimental motion capture trials. The model inputs of IPA were kinetics variables of gravity and all joint moments, which were calculated from inverse dynamics. After the model was configured, gravity and all joint moments were set to zero. One joint moment was then entered into the compiled MATLAB function that allowed calculation of the accelerations at all joints due solely to the input moment. The joint moment was then reset to zero, the model was reset to the initial configuration, and another joint moment or gravity (from the same frame of the motion capture data) was sequentially fed into this compiled MATLAB function. This process was repeated for each frame of data collected during the reaching phase. The model output provided the accelerations at all joints that were generated by each input joint moment or gravity. In order to get IPA output, the double integrator was created as a MATLAB function to integrate induced accelerations, then integrated with induced velocity to reconstruct induced position.

\section{Statistical Analysis}

The Kolmogorov-Smirnov test was used to examine the normality of the dependent variables. The statistical significance level for all comparisons was set at $p<0.05$. One-way repeated measures analysis of variance (parametric statistics) or Wilcoxon signed ranks test (two related samples, nonparametric statistics) were used to analyze the dependent variables in the paretic arm between bilateral reaching task and unilateral reaching task depending on the results of the KolmogorovSmirnov test. The relationships between the compensatory movement control strategy (predictors) and functional measures (outcome measures) were determined using Pearson correlations with baseline FM scores and postbaseline change scores for WT and UMAQS. For these analyses, the contribution of shoulder moment and elbow moment on hand position trajectory were used as predictors.

\section{RESULTS}

\section{Compensatory Movement Strategies}

There were no significant differences between unilateral and bilateral reaching tasks in contributions of shoulder, elbow, wrist moment, and gravity (Wilcoxon test, $p>$ 0.05 ), although contribution of the shoulder moment and elbow moment to hand position were both slightly reduced in the unilateral task (Figure 2(a)). Therefore, the tasks were combined in Figure 2(b) for all 12 participants. Shoulder moment was the largest contributor to the hand forward reaching trajectory $(1.221 \pm 0.081 \mathrm{~m})$. Elbow moment was the largest contributor to the hand backward (or slowing of the) reaching trajectory $(-1.31 \pm$ $0.078 \mathrm{~m}$ ). These results differ from nondisabled adults, in whom the shoulder moment was larger than the elbow moment [23]. In both sets of results, the pattern of hand position induced by the net moment indicated that the shoulder moment was a major mover of the arm, which "intended" to move the hand beyond the target, called an 
"overshooting" effect. Similarly, the elbow moment was a prime mover of the arm, which "intended" to move the hand short of the target, called an "undershooting" effect because the elbow has to make the dual effort to flex the elbow and extend the shoulder, which drives the arm to undershoot the target. The shoulder and elbow essentially work against each other to make sure the hand can reach the target. This control synergy between shoulder and elbow has been termed a "directional control principle" [23]. This relative reduction of shoulder moment in the individuals with stroke was compensated for by small contributions

(a)

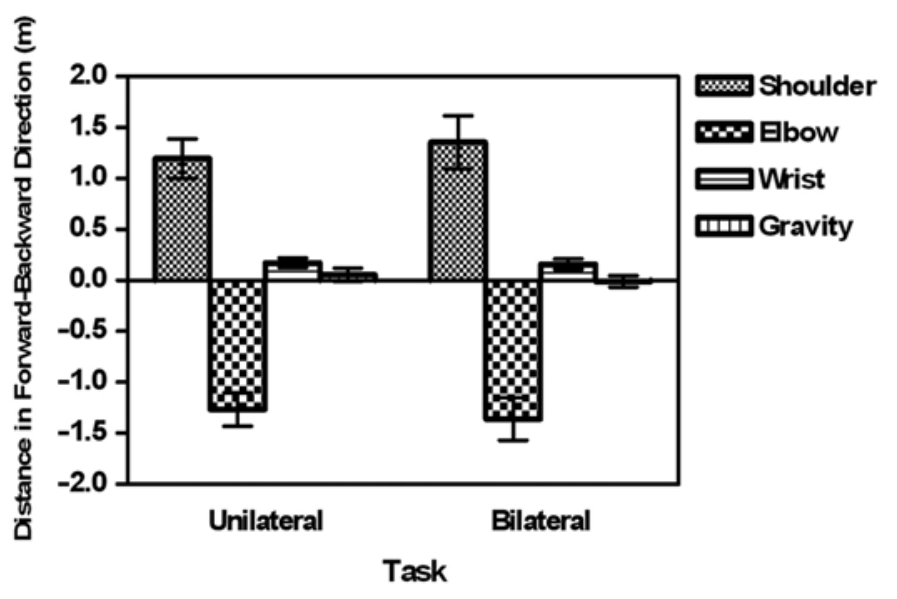

(b)

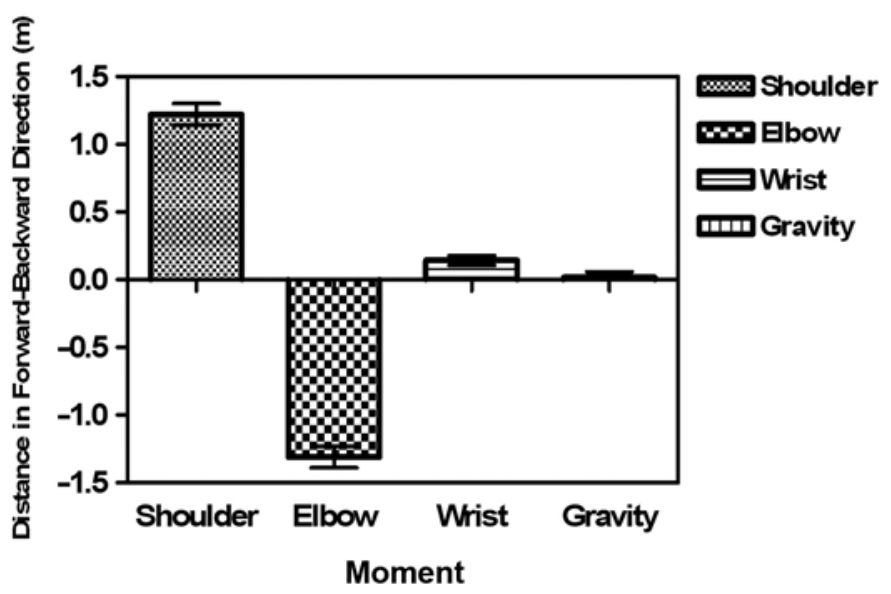

Figure 2.

(a) Bar graph of final induced hand position from net moment acting on shoulder, elbow, wrist, and gravity in unilateral and bilateral reaching tasks. (b) Bar graph of final induced hand position from net moment acting on shoulder, elbow, wrist, and gravity across unilateral and bilateral reaching tasks. from the wrist moment and gravity. A large variability of wrist and gravity contributors between subjects was observed (wrist: $0.143 \pm 0.037 \mathrm{~m}$, range: $0.216 \sim 0.071 \mathrm{~m}$; gravity: $0.015 \pm 0.043 \mathrm{~m}$, range: $0.099 \sim 0.069 \mathrm{~m}$ ), indicating that as a group the results do not reflect a common compensatory control strategy.

\section{Subset Analysis: Compensatory Movement Strategy Based on Impairment Level}

The 12 participants fall into two clusters based on baseline FM score. Of the 12 participants, 9 fell into an FM score range between 6 and 23, and 3 of 12 participants fell into an FM score range between 38 and 53. These groups can be classified as $\leq 25$ (severe) or $>25$ (mild to moderate). In addition to the observed natural clustering, a score of 25 has previously been used as a delimitation mark for severe impairment [8]. A Mann-Whitney U-test (two independent samples, nonparametric statistics) was used to analyze the dependent variables of shoulder and elbow moment contributions in the paretic arm between the severe and mild to moderate impairment groups.

Across tasks, contributions of shoulder, elbow, wrist moment, and gravity to the hand trajectory in forwardbackward direction in severe and mild to moderate impaired groups are shown in Figure 3. The two groups illustrated different movement control strategies. Elbow moment contribution in the severe impaired group $(-1.41 \pm$ $0.098 \mathrm{~m}$ ) was significantly higher than in the mild to

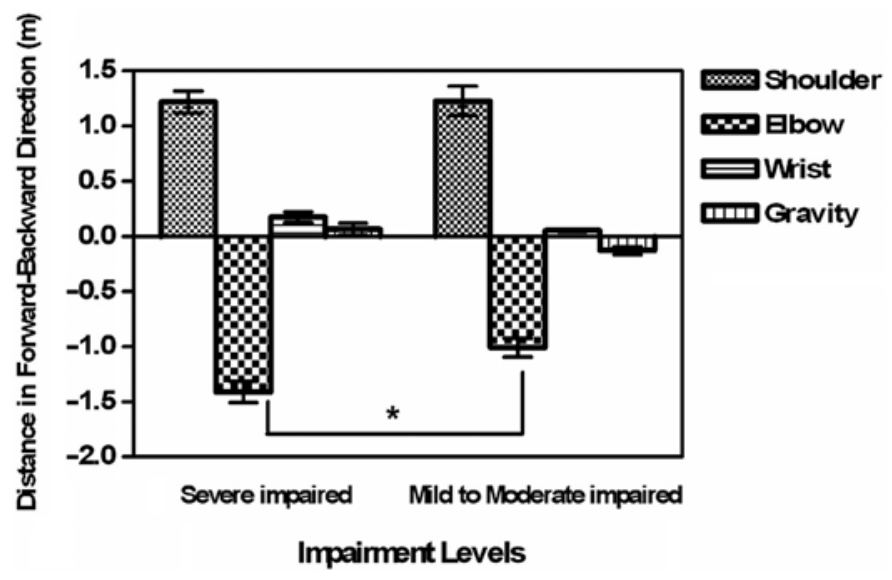

Figure 3.

Bar graph of final induced hand position from net moment acting on shoulder, elbow, wrist, and gravity in severe and mild to moderate impaired groups. "Significant difference between "severe" and "mild to moderate" impaired group at elbow moment contribution $(p<0.05)$. 
moderate impaired group $(-1.01 \pm 0.085 \mathrm{~m}$, Mann-Whitney U-test, $p=0.03$ ). There was no significant difference in shoulder moment contribution between the two groups $(p=$ $0.75)$. Gravity contribution was higher in the severe impaired group $(0.064 \pm 0.055 \mathrm{~m})$, where it contributed to forward movement, than in the mild to moderate impaired group $(-0.13 \pm 0.038 \mathrm{~m})$, where it contributed to backward slowing movement, but there was no significant difference between the two subgroups (Mann-Whitney U-test, $p=$ $0.34)$.

\section{Clinical Correlations}

Following progressive BATRAC training, the baseline shoulder moment contribution (of all subjects) was correlated with an improvement in WT ( $r=-0.468, p=0.001)$ using individual trials (Figure 4). Elbow moment contribution did not correlate with the WT improvement, and neither shoulder nor elbow moment contributions correlated with the UMAQS improvement $(p>0.05)$. Not all participants improved their WT; therefore, we conducted another subanalysis to determine whether movement compensatory strategies were different based on actual improvement in WT and also whether this was related to their initial impairment level.

\section{Subset Analysis: Compensatory Movement Strategy Based on WT Outcome}

Posttraining results indicated that 5 of the 12 participants showed improvement in this measure. The participants were categorized as showing a "good" outcome if

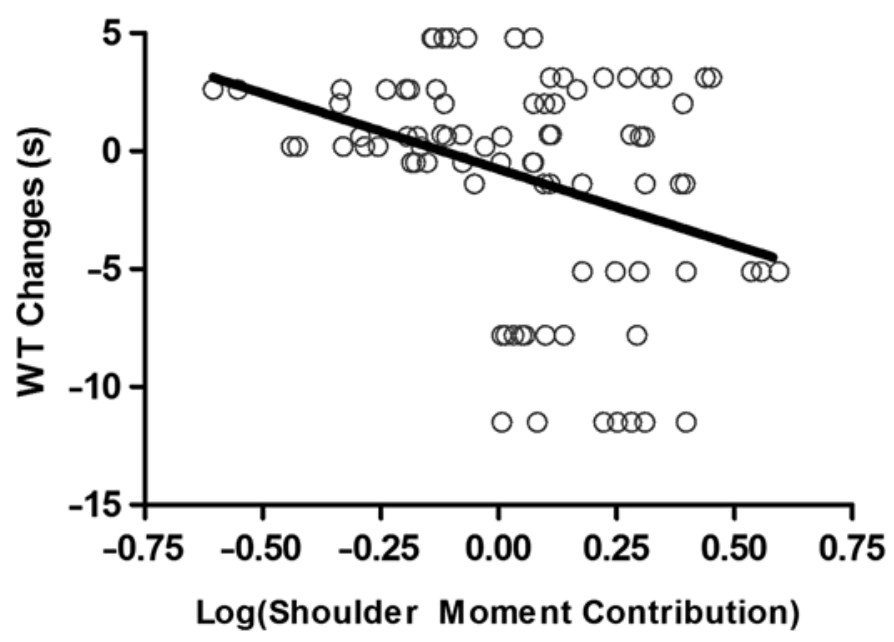

Figure 1.

Correlation between shoulder moment contribution and improvement in Modified Wolf Motion Function Test (WT) score. the WT posttraining was shorter than pretraining and a "poor" outcome if the WT posttraining was longer than pretraining. A Mann-Whitney U-test (two independent samples, nonparametric statistics) was used to analyze the dependent variables in the paretic arm between the poor and good outcome groups. Contributions of shoulder, elbow, wrist moment, and gravity to hand trajectory in forward-backward directions in the good and poor outcome groups are shown in Figure 5. Significant differences existed in shoulder moment and gravity contributions between the good $(1.638 \pm 0.136 \mathrm{~m})$ and poor $(0.901 \pm$ $0.0687 \mathrm{~m}$ ) outcome groups in the combined bilateral and unilateral reaching tasks (Mann-Whitney U-test, $p<0.05$ ). Significant reduction of shoulder moment contribution in the poor outcome group was compensated for by a significant increased "use" of gravity compared with the good outcome group. Interestingly, the five participants in the good outcome group were distributed into two different impairment groups. Three in the severe impaired group and two in the mild to moderate impaired group showed improvement in WT, which is in line with the suggestion that impairment level, by itself, may not predict treatment outcome.

We found no significant differences for wrist and elbow moment contributions between the good outcome and poor outcome groups. Therefore, it is possible that different participants reached the same target location using different angular motions because the compensatory

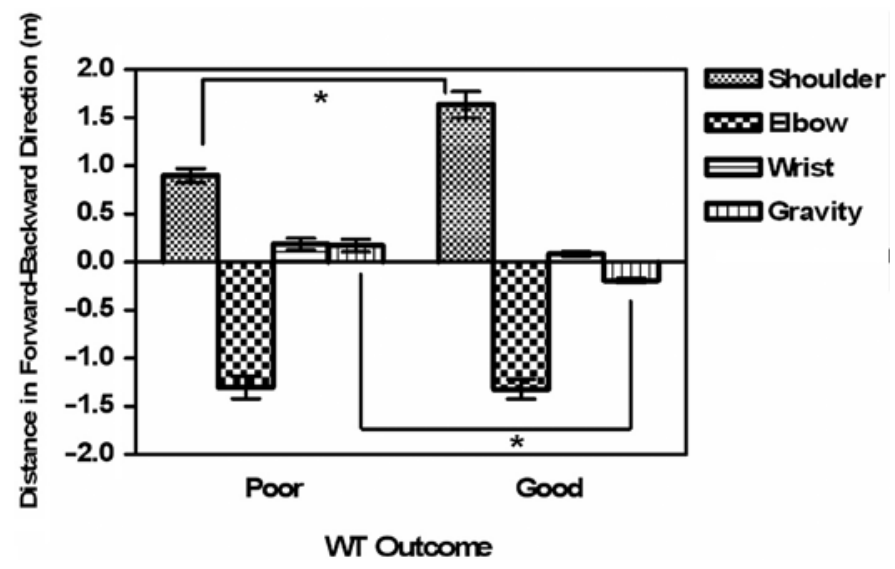

Figure 4.

Bar graph of final induced hand position from net moment acting on shoulder, elbow, wrist, and gravity good and poor outcome groups. WT $=$ Modified Wolf Motor Function Test. "Significant difference between "good" and "poor" outcome group at shoulder moment and gravity contribution $(p<0.05)$. 
strategies captured using IPA were based on the summation of effort across individual joint moments to determine the contributing factors of hand position during arm reaching. Furthermore, IPA also does not inform us of the angular segmental positions at the end of reaching. To address these potential sources of variance, we determined the forearm angular kinematics (elbow Euler joint angle in z-direction) based on the functional outcomes. We found that the good outcome group $\left(44.71 \pm 12.96^{\circ}\right)$ did indeed have significantly larger forearm supination of the paretic arm compared with the poor outcome group (34.23 $\left.\pm 23.10^{\circ}\right)$ (Mann-Whitney U-test, $p<0.01$ ).

\section{DISCUSSION}

This study quantified compensatory movement strategies of paretic arm reaching in chronic stroke survivors using the tool of IPA. The overall pattern of arm control strategy was characterized by a greater contribution of the elbow moment slowing movement during arm reaching in comparison to the shoulder moment's contribution to the forward reach, which included some contributions of wrist and gravity. There were no differences of strategy between reaching unilaterally and bilaterally, but an increased shoulder relative to elbow moment contribution was associated with less impairment and with greater gains of speed in functional tasks. Level of impairment did not, by itself, correlate with training gains, and control strategy differences did not correlate with gains in activities of daily living.

The predominant movement control strategy for the individuals with chronic stroke only partially mirrors that of nondisabled individuals. When the arm reaches out in the forward direction, the shoulder and elbow contribute to the hand position in two different directions, moving the arm forward and counteracting this movement, the so-called directional control principle [23]. Stroke survivors also showed patterns consistent with the directional control principle, but the use of contributors was different because the shoulder moment was less than the elbow moment compared with nondisabled adults. We demonstrate that gravity and the wrist are also recruited to compensate for the reduced shoulder moment contribution in order to satisfy the directional control principle. This reduction of shoulder moment contribution relative to elbow moment causes the arm to situate in a flexed position during the middle phase of arm reaching as a result of a flexor moment about the elbow during the reaching phase. During the middle phase of arm reaching, the forearm supinates and the elbow flexor moment moves the hand away from the box vertically. Gravity plays a passive counterbalance with the elbow moment and makes the hand drop down to the table as it reaches forward to touch the box. With reference to other descriptions of compensatory movement strategies, the IPA analysis emphasizes not only a relative reduction in contribution of the shoulder versus the elbow but also the use made of the wrist moment and gravity in compensation.

Contrary to our expectation, we did not find any significant difference in compensatory movement strategies between unilateral and bilateral reaching tasks. Previous results on the bilateral benefit of stroke arm reaching were based on an end-point trajectory while the task was performed at fast speed [17]; our results were based on a preferred speed reaching task, which is more consistent with the nature of a functional task in daily life. It is plausible that a maximum speed task would elicit differences in the direction of a larger shoulder moment contribution for bilateral activities. If our speculation were true, this would support the idea that more intense bilateral training progressing on speed may be better than bilateral training at a comfortable speed.

Our subset analysis results based on impairment levels suggest that compensatory moment strategies are related to the degree of impairment. This result is consistent with Levin's kinematic analysis, which showed recruiting a new degree of freedom (trunk) is strongly correlated with a higher impairment level [11-12]. In the present case, the moderate to mild impairment group had similar contribution patterns to nondisabled adults [23], in whom the shoulder contribution was larger than that of the elbow moment, while in the severe impairment group the elbow moment contribution to hand position was larger than that of the shoulder moment. Therefore, one clinical strategy for patients with severe impairment levels would be to assess and concentrate on improving the shoulder moment. An alternative or complementary strategy would be to try to reduce the elbow flexor moment such that it contributes less or at least more equally to the shoulder forward moments.

Compensatory movement strategies also correlated with posttraining functional changes, but only on the WT (Figure 4). Participants who had more shoulder moment contribution relative to elbow contribution had a greater benefit to the paretic arm as a result of bilateral arm training 
as measured by improvement of speed during WT. This result also supports working on strategies for improving the shoulder moment contribution, particularly if the shoulder moment is thought to be less than the elbow moment. For example, Dewald and Beer found that there was abnormal coupling between shoulder and elbow joint torque in the unsupported condition (gravity not eliminated) [31]. Increasing the shoulder moment contribution could benefit the abnormal torque coupling seen after stroke because, if the shoulder could be flexed more with reduced shoulder abduction, this may reduce the relatively larger elbow flexion torque.

The fact that high shoulder relative to elbow moment contribution is related to both low impairment and improvement after training is significant since low impairment by itself did not predict improvement. This result suggests that the movement strategies identified here may be more sensitive than an overall impairment level in predicting a response after this type of training. On the other hand, we did not find a significant correlation between compensatory movement strategies and UMAQS. The UMAQS is a clinical assessment in the disability domain that measures daily use of the paretic arm in unilateral and bilateral tasks. As such, maybe the relationship between the elements of the fundamental movement strategies identified here and the regaining of skills used in daily life is not as compatible as regaining the ability to move an existing skill faster, as found in the WT result. Certainly, the present result is consistent with a study that found no correlation between initial grip strength and posttraining scores on the UMAQS, albeit after a different training regimen [27]. There must be other factors that influence the regaining of skills used in daily life. This finding supports an argument that we should not rely on unidimensional assessments in intervention studies.

One characteristic of the IPA analysis is that it accounts for only reaching forward in a linear translation and not for a rotation effect of the forearm as it supinates. All participants showed some forearm supination during the middle and terminal parts of the reach. In an ideal, nondisabled participant, using our experimental task, there would be $80^{\circ}$ to $90^{\circ}$ of forearm supination in order to achieve the reaching goal as requested. In a small subset of participants who were not from this study, we found that the wrist joint had an abnormal abductor moment associated with forearm supination [32]. This abnormal abductor moment made it difficult for the wrist joint to counterbalance gravity because the forearm supi- nation had a rotational effect on the wrist joint from the flexion-extension direction to the abduction direction. In the present analysis, our results indicate that the participants who had good functional outcomes not only had larger relative shoulder moment contributions but also had a larger range of motion during forearm supination. In the "poor" outcome group, the effect of gravity to drop the arm passively was increased to compensate for the reduction in shoulder moment contribution that moved the arm forward as well as the lack of ability to supinate the forearm that would also work against gravity. In other words, these subjects used gravity to help them accomplish the task. Clinically, then, it might also be important to work on regaining supination of the forearm since this will allow better stabilization and function by the wrist and hand in a reaching task.

Overall, the use of the IPA approach in this study has provided several clinical implications through the biomechanical assessment of compensatory movement strategies. For example, this approach can be incorporated with other clinical tests for more complete assessment of the effectiveness of intervention. Functional clinical tests tend to measure the success of accomplishing the task but do not quantify the compensatory movement strategy. Similarly, while current neurorehabilitation interventions are focused on maximizing the patient's functional outcome, optimizing or changing someone's usage of compensatory movement strategies may be associated with the recovery process. For example, interventions could focus on reduction of abnormal coupling between shoulder and elbow by increasing shoulder moment contribution or decreasing elbow moment contribution. This would optimize the relative contribution between shoulder and elbow. Finally, arm reaching performance relies on multijoint coordination, but many clinical tests are focusing on a single joint. The IPA approach provides information on the role of individual joints during a multijoint movement. This information may be useful for clinicians to make a better judgment of patients' motor performance during multijoint tasks such as arm reaching.

There are three limitations of this study. One limitation is the small sample size, particularly for the subset analysis. For the most part, however, we were able to detect differences with a small sample size. A second limitation is that the IPA approach in this article was based on total effort of net moments from individual joints. It is possible that summation effects do not detect the contribution of each mechanical degree of freedom. For example, it is 
plausible that the shoulder flexion-extension moment may play a different role than shoulder abduction-adduction moment during arm reaching. However, motion capture data from this study did not indicate a major role of shoulder abduction-adduction moments associated with hand position. Also, the IPA analysis provided information on the final ending position determined by all contributors, but it did not describe differences that occurred at different time phases of arm reaching. A third limitation is that the trunk was fixed as a ground in the current model. Given that we restricted trunk movement, the compensatory strategies identified should be interpreted within this specific situation, akin to many training situations, and cannot be generalized necessarily to unconstrained situations. It will be important to add the trunk segment into the model because the two arms are linked with the trunk and there are potential contributing influences from one limb to the other across the trunk. Future work will focus on developing a forward dynamics model that includes the unconstrained trunk and identifying contributors from each degree of freedom. We will also determine whether the intervention affects the compensatory strategies and assess the effects of changing speed.

\section{CONCLUSIONS}

In conclusion, the IPA analysis demonstrated that the compensatory movement control strategies after stroke result in a lower than typical shoulder moment contribution, along with a higher than typical elbow moment contribution. The shoulder moment contribution was compensated for by increasing wrist and gravity contributions. The compensatory strategies are related to impairment level as well as speed of functional movement outcomes after bilateral training.

\section{ACKNOWLEDGMENTS}

\author{
Author Contributions: \\ Study concept and design: W. Liu, J. Whitall. \\ Acquisition of data: W. Liu. \\ Analysis and interpretation of data: W. Liu, T. M. Kepple, J. Whitall, \\ S. McCombe Waller. \\ Drafting of manuscript: W. Liu. \\ Critical revision of manuscript for important intellectual content: \\ T. M. Kepple, J. Whitall, S. McCombe Waller. \\ Statistical analysis: W. Liu. \\ Obtained funding: J. Whitall.
}

Administrative, technical, or material support: J. Whitall, T. M. Kepple. Study supervision: J. Whitall.

Financial Disclosures: The authors have declared that no competing interests exist.

Funding/Support: This material was based on work supported by the National Institute on Aging Claude D. Pepper Older Americans Independence Center (grant P60-AG12583; A. P. Goldberg, principal investigator [PI]), and by the National Institute on Disability and Rehabilitation Research (grant H133G010111; J. Whitall, PI).

Additional Contributions: Dr. Liu is now with the Department of Physical Therapy, College of Allied Health, University of South Alabama, Mobile, Alabama.

Institutional Review: All participants provided written informed consent approved by both the University of Maryland and Baltimore Veterans Affairs Medical Center Institutional Review Boards.

Participant Follow-Up: The authors do not plan to inform participants of the publication of this study. However, participants have been encouraged to check the study Web site for updated publications.

\section{REFERENCES}

1. Rosamond W, Flegal K, Friday G, Furie K, Go A, Greenlund K, Haase N, Ho M, Howard V, Kissela B, Kittner S, Lloyd-Jones D, McDermott M, Meigs J, Moy C, Nichol G, O’Donnell CJ, Roger V, Rumsfeld J, Sorlie P, Steinberger J, Thom T, Wasserthiel-Smoller S, Hong Y; American Heart Association Statistics Committee and Stroke Statistics Subcommittee. Heart disease and stroke statistics2007 update: a report from the American Heart Association Statistics Committee and Stroke Statistics Subcommittee. Circulation. 2007;115(5):e69-171. [PMID:17194875] http://dx.doi.org/10.1161/CIRCULATIONAHA.106.179918

2. Williams GR. Incidence and characteristics of total stroke in the United States. BMC Neurol. 2001;1:2.

[PMID:11446903]

http://dx.doi.org/10.1186/1471-2377-1-2

3. Gowland C, deBruin H, Basmajian JV, Plews N, Burcea I. Agonist and antagonist activity during voluntary upperlimb movement in patients with stroke. Phys Ther. 1992; 72(9):624-33. [PMID:1508970]

4. Taub E, Crago JE, Burgio LD, Groomes TE, Cook EW 3rd, DeLuca SC, Miller NE. An operant approach to rehabilitation medicine: overcoming learned nonuse by shaping. J Exp Anal Behav. 1994;61(2):281-93. [PMID:8169577] http://dx.doi.org/10.1901/jeab.1994.61-281

5. Krebs HI, Hogan N, Volpe BT, Aisen ML, Edelstein L, Diels C. Overview of clinical trials with MIT-MANUS: a robot-aided neuro-rehabilitation facility. Technol Health Care. 1999;7(6):419-23. [PMID:10665675]

6. Burgar CG, Lum PS, Shor PC, Machiel Van der Loos HF. Development of robots for rehabilitation therapy: the Palo Alto VA/Stanford experience. J Rehabil Res Dev. 2000; 37(6):663-73. [PMID:11321002] 
7. Whitall J, McCombe Waller S, Silver KH, Macko RF. Repetitive bilateral arm training with rhythmic auditory cueing improves motor function in chronic hemiparetic stroke. Stroke. 2000;31(10):2390-95. [PMID:11022069] http://dx.doi.org/10.1161/01.STR.31.10.2390

8. Luft AR, McCombe-Waller S, Whitall J, Forrester LW, Macko R, Sorkin JD, Schulz JB, Goldberg AP, Hanley DF. Repetitive bilateral arm training and motor cortex activation in chronic stroke: a randomized controlled trial. JAMA. 2004;292(15):1853-61. [PMID:15494583] http://dx.doi.org/10.1001/jama.292.15.1853

9. Levin MF, Kleim JA, Wolf SL. What do motor "recovery" and "compensation" mean in patients following stroke? Neurorehabil Neural Repair. 2009;23(4):313-19. [PMID:19118128] http://dx.doi.org/10.1177/1545968308328727

10. Brunnström S. Movement therapy in hemiplegia: A neurophysiological approach. Philadelphia (PA): Harper and Row; 1970.

11. Levin MF. Interjoint coordination during pointing movements is disrupted in spastic hemiparesis. Brain. 1996; 119(Pt 1):281-93. [PMID:8624689] http://dx.doi.org/10.1093/brain/119.1.281

12. Roby-Brami A, Feydy A, Combeaud M, Biryukova EV, Bussel B, Levin MF. Motor compensation and recovery for reaching in stroke patients. Acta Neurol Scand. 2003; 107(5):369-81. [PMID:12713530] http://dx.doi.org/10.1034/j.1600-0404.2003.00021.x

13. McCrea PH, Eng JJ, Hodgson AJ. Saturated muscle activation contributes to compensatory reaching strategies after stroke. J Neurophysiol. 2005;94(5):2999-3008. [PMID:16014786] http://dx.doi.org/10.1152/jn.00732.2004

14. Kelso JA, Southard DL, Goodman D. On the coordination of two-handed movements. J Exp Psychol Hum Percept Perform. 1979;5(2):229-38. [PMID:528935] http://dx.doi.org/10.1037/0096-1523.5.2.229

15. Swinnen SP, Young DE, Walter CB, Serrien DJ. Control of asymmetrical bimanual movements. Exp Brain Res. 1991; 85(1):163-73. [PMID:1884755] http://dx.doi.org/10.1007/BF00229998

16. Harris-Love ML, McCombe Waller S, Whitall J. Exploiting interlimb coupling to improve paretic arm reaching performance in people with chronic stroke. Arch Phys Med Rehabil. 2005;86(11):2131-37. [PMID:16271560] http://dx.doi.org/10.1016/j.apmr.2005.05.006

17. McCombe Waller S, Harris-Love M, Liu W, Whitall J. Temporal coordination of the arms during bilateral simultaneous and sequential movements in patients with chronic hemiparesis. Exp Brain Res. 2006;168(3):450-54.

[PMID:16331507] http://dx.doi.org/10.1007/s00221-005-0235-3
18. Zajac FE, Gordon ME. Determining muscle's force and action in multi-articular movement. Exerc Sport Sci Rev. 1989;17:187-230. [PMID:2676547]

19. Lang CE, Beebe JA. Relating movement control at 9 upper extremity segments to loss of hand function in people with chronic hemiparesis. Neurorehabil Neural Repair. 2007; 21(3):279-91. [PMID:17353458] http://dx.doi.org/10.1177/1545968306296964

20. Kepple TM, Siegel KL, Stanhope SJ. Relative contributions of the lower extremity joint moments to forward progression and support during stance. Gait Posture. 1997; 6:1-8. http://dx.doi.org/10.1016/S0966-6362(96)01094-6

21. Fregly BJ, Zajac FE. A state-space analysis of mechanical energy generation, absorption, and transfer during pedaling. J Biomech. 1996;29(1):81-90. [PMID:8839020] http://dx.doi.org/10.1016/0021-9290(95)00011-9

22. Anderson FC, Goldberg SR, Pandy MG, Delp SL. Contributions of muscle forces and toe-off kinematics to peak knee flexion during the swing phase of normal gait: an induced position analysis. J Biomech. 2004;37(5):731-37. [PMID:15047002] http://dx.doi.org/10.1016/j.jbiomech.2003.09.018

23. Liu W, Whitall J, Kepple T. Multi-joint coordination of functional arm reaching: induce position analysis. J Appl Biomech. 2012. [Epub ahead of print]. [PMID:22814206]

24. Chen SY, Winstein CJ. A systematic review of voluntary arm recovery in hemiparetic stroke: critical predictors for meaningful outcomes using the international classification of functioning, disability, and health. J Neurol Phys Ther. 2009;33(1):2-13. [PMID:19265766]

25. Fugl-Meyer AR, Jääskö L, Leyman I, Olsson S, Steglind S. The post-stroke hemiplegic patient. 1. a method for evaluation of physical performance. Scand J Rehabil Med. 1975; 7(1):13-31. [PMID:1135616]

26. Whitall J, Savin DN Jr, Harris-Love M, Waller SM. Psychometric properties of a modified Wolf Motor Function test for people with mild and moderate upper-extremity hemiparesis. Arch Phys Med Rehabil. 2006;87(5):656-60. [PMID:16635628] http://dx.doi.org/10.1016/j.apmr.2006.02.004

27. Bovend'Eerdt TJ, Dawes H, Johansen-Berg H, Wade DT. Evaluation of the Modified Jebsen Test of Hand Function and the University of Maryland Arm Questionnaire for Stroke. Clin Rehabil. 2004;18(2):195-202. [PMID:15053129] http://dx.doi.org/10.1191/0269215504cr722oa

28. Wolf SL, Catlin PA, Ellis M, Archer AL, Morgan B, Piacentino A. Assessing Wolf motor function test as outcome measure for research in patients after stroke. Stroke. 2001; 32(7):1635-39. [PMID:11441212] http://dx.doi.org/10.1161/01.STR.32.7.1635

29. Morris DM, Uswatte G, Crago JE, Cook EW 3rd, Taub E. The reliability of the wolf motor function test for assessing 
upper extremity function after stroke. Arch Phys Med Rehabil. 2001;82(6):750-55. [PMID:11387578]

http://dx.doi.org/10.1053/apmr.2001.23183

30. Winter DA. Biomechanics and motor control of human movement. 3rd ed. New York (NY): John Wiley \& Sons; 2005.

31. Dewald JP, Beer RF. Abnormal joint torque patterns in the paretic upper limb of subjects with hemiparesis. Muscle Nerve. 2001;24(2):273-83. [PMID:11180211] http://dx.doi.org/10.1002/1097-4598(200102)24:2<273::AIDMUS130>3.0.CO;2-Z

32. Liu W, Rodgers MM, McCombe Waller S, Kepple T, Whitall J. Upper extremity kinetic model of functional arm reaching in stroke. Proceedings of the 33th Annual Meeting of the American Society of Biomechanics; 2009; University Park, PA.
Submitted for publication April 18, 2011. Accepted in revised form May 23, 2012.

This article and any supplementary material should be cited as follows:

Liu W, McCombe Waller S, Kepple TM, Whitall J. Compensatory arm reaching strategies after stroke: Induced position analysis. J Rehabil Res Dev. 2013;50(1):71-84. http://dx.doi.org/10.1682/JRRD.2011.04.0074

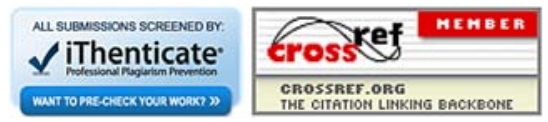


